\title{
Nurse supply grows despite shortage fears
}

$\mathrm{C}$

ontrary to concerns about a shortage in Canada, the number of regulated nurses across the country has continued to rise in recent years, finds a new Canadian Institute for Health Information (CIHI) report.

According to Regulated Nurses, 2013, there were more than 408000 registered nurses, nurse practitioners and licensed practical nurses eligible to practise in Canada last year. That represents an $8 \%$ increase in the nurse supply since 2009, outpacing growth in both the Canadian labour force and general population over the same period.

"We continue to hear about issues from employers who can't fill vacancies and nurses who are overwhelmed," says Andrea Porter-Chapman, a CIHI health human resources specialist. "But from the macro level, we are seeing the growth continuing and, in recent years, increasing."

Canada's supply of nurses increased at an average annual rate of $2.1 \%$ between 2009 and 2013, up from 1.8\% between 2003 and 2008. Last year, the number of new nurses also outpaced those retiring from the profession, resulting in a net gain of 8173 nurses.

Licensed practical nurses (LPNs) have seen the greatest influx. The LPN supply has grown by more than $50 \%$ since 2003, while the supply of nurse practitioners has grown by $15 \%$ and registered psychiatric nurses by $8 \%$. More than half of the current LPN workforce is younger than 40 .

However, this group also was less likely to be employed at the time of registration compared to other nurses. In addition, LPNs took longer to find full-time employment after graduation, waiting up to 10 years compared to just five for registered nurses and registered psychiatric nurses.

Porter-Chapman say this may be due in part to retention policies put in place by employers and ministries in

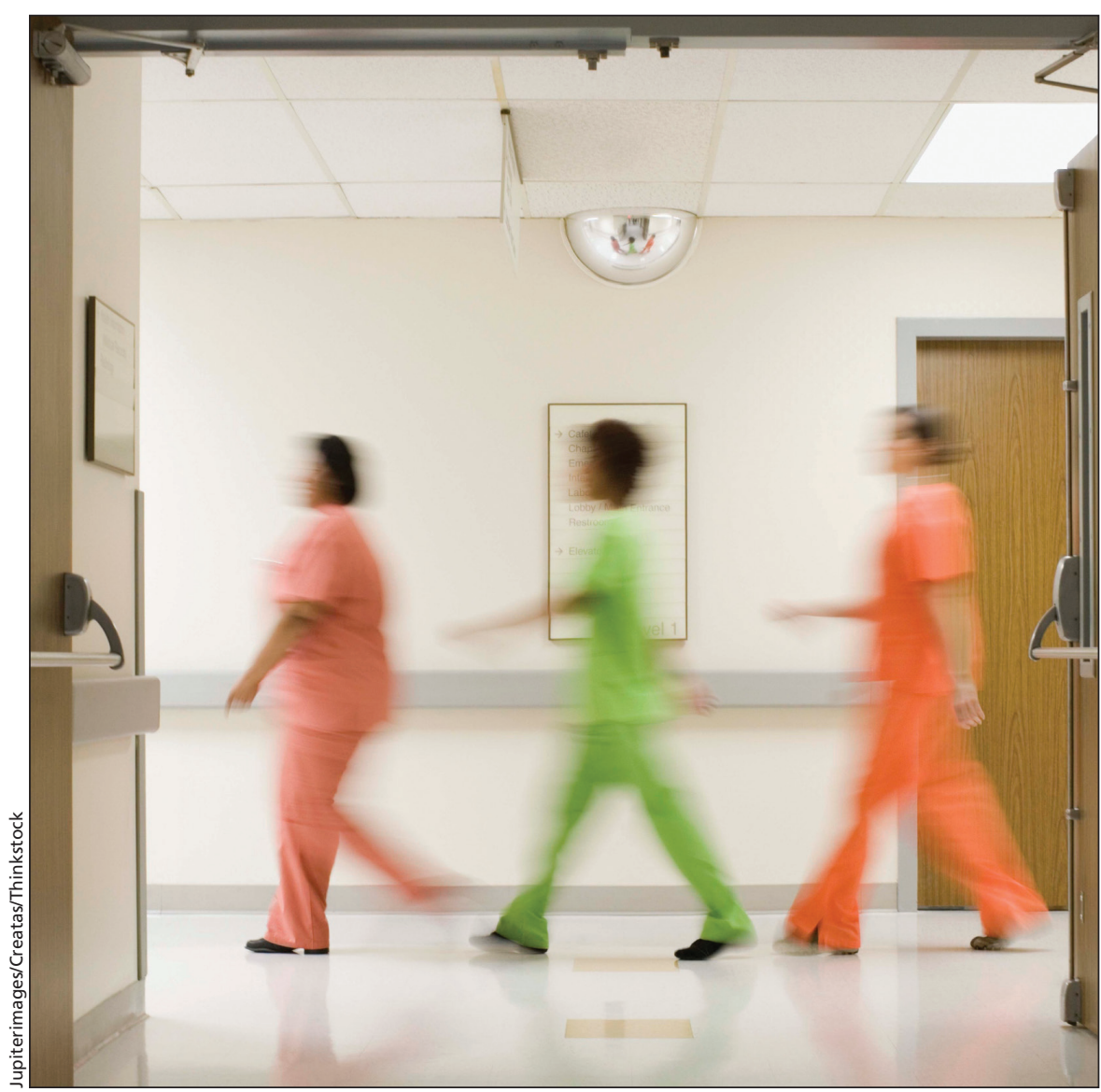

Growth in Canada's supply of nurses is continuing and accelerating, according to the Canadian Institute for Health Information.

the 1990s and early 2000s to stave off possible shortages.

"Now we're seeing those older nurses working part-time, partly because of the retention strategies; meanwhile we have more LPNs coming in but only part-time positions available to them," she explains.

Although, "that's not to say a nurse might not be working two or three positions to the equivalent of a full-time position," a factor not considered by CIHI's study.

The data suggest it's time to start looking beyond "just the numbers" when talking about shortages, says PorterChapman. "We've seen the scopes of practice shifting and what the roles are for LPNs, and that's something that needs to be explored in every facility."

Karima Velji, president of the Canadian Nurses Association, agrees that the "numbers tell just a portion of the story."

"Canada will only realize meaningful improvements in population health and system performance when its nurses are employed full-time in positions that enable them to practise to their full scope of education and skills and where they can best meet the health needs of our communities," she said in a statement. — Lauren Vogel, CMAJ

CMAJ 2014. DOI:10.1503/cmaj.109-4848 